\title{
HORMONES, OSCILLATIONS ET VAGUES CALCIQUES
}

\section{Michel Claret Jean-Pierre Mauger}

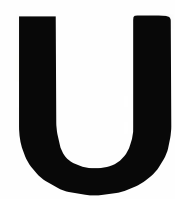

ne observation inattendue de ces dernières années est qu'une variété d'hormones circulantes provoquent, dans leurs cellules cibles, non pas des montées plus ou moins soutenues de la concentration du $\mathrm{Ca}^{2+}$ cytosolique $\left(\left[\mathrm{Ca}^{2+}\right]_{i}\right.$, mais de larges fluctuations du $\left[\mathrm{Ca}^{2+}\right]_{i}$ appelées oscillations calciques [1]. Ces oscillations sont souvent associées à la propagation de vagues calciques, qui naissent au niveau de la membrane plasmique, puis se propagent dans la cellule. A ce jour, ces oscillations et ces vagues apparaissent être impliquées dans un nombre considérable de réponses cellulaires: métabolisme, sécrétion, contraction, agrégation, fécondation, photoréception et prolifération.

Comment et pourquoi naissent ces oscillations et ces vagues calciques, quels sont les mécanismes intracellulaires impliqués, et quels moyens techniques permettent de les mettre en évidence, sont les questions discutées dans cet Éditorial et dans l'article de C. Pavoine et F. Pecker. Pour des raisons de survie, toutes les cellules animales dépensent continuellement de l'énergie pour maintenir leur $\left[\mathrm{Ca}^{2+}\right]_{\text {i }}$ à un niveau très faible $(0,1 \mu \mathrm{M})$. Les cellules utilisent plusieurs systèmes pour se prémunir contre un envahissement du $\mathrm{Ca}^{2+}$ plasmatique: (1) au niveau de la membrane plasmique, par le maintien d'une perméabilité de repos basse, et par un rejet continu du $\mathrm{Ca}^{2+}$ cytosolique vers le plasma; (2) au niveau du cytosol, par la liaison du $\mathrm{Ca}^{2+}$ sur de multiples protéines, de type calmoduline, par exemple, et par une accumulation du $\mathrm{Ca}^{2+}$ dans la lumière du réticulum endoplasmique. Dans une moindre mesure, le $\mathrm{Ca}^{2+}$ est également concentré dans l'espace périnucléaire et les mitochondries. L'abaissement de la $\left[\mathrm{Ca}^{2+}\right]_{i}$ à un niveau très bas présente un avantage supplémentaire. Il permet à la cellule de créer des gradients de concentration calcique très élevés avec le plasma et avec la lumière du réticulum endoplasmique, condition requise pour déclencher des variations très rapides de la $\left[\mathrm{Ca}^{2+}\right]_{i}$. Cela explique pourquoi le $\mathrm{Ca}^{2+}$ est universellement utilisé comme système signal, non seulement dans les réponses cellulaires lentes comme la libération de glucose dans l'hépatocyte, l'agrégation plaquettaire ou la fécondation, mais aussi dans les phénomènes beaucoup plus rapides telles la libération de neurotransmetteurs ou la contraction musculaire [2].

L'activation des récepteurs cellulaires par des hormones entaîne une libération de $\mathrm{Ca}^{2+}$ intracellulaire, par activation d'un récepteur-canal localisé dans la membrane du réticulum endoplasmique, et stimulation du flux entrant de $\mathrm{Ca}^{2+}$ plasmatique. La transmission du signal reçu par la membrane est assurée par l'hydrolyse d'un phospholipide, le 
phosphatidylinositol 4,5-bisphosphate, via l'activation d'une phospholipase C- $\beta$ et de protéines trimériques à activité GTPasique. En 1983, M. Berridge découvrait que le produit de l'hydrolyse du phospholipide, l'inositol $(1,4,5)$-trisphosphate $\left(\mathrm{InsP}_{3}\right)$, était le messager intracellulaire responsable de la mobilisation du $\mathrm{Ca}^{2+}$ depuis le réticulum endoplasmique. En ouvrant le récepteurcanal, l'InsP $\mathrm{P}_{3}$ libère le $\mathrm{Ca}^{2+}$ concentré dans l'organite. L'autre second messager produit, le diacylglycérol, active des protéine kinases $C$ qui jouent des rôles clefs dans les réponses cellulaires. Les facteurs de croissance stimulent également la production de l'InsP ${ }_{3}$ en activant des récepteurs à activité tyrosine-kinase, qui phosphorylent et activent une phospholipase C-y. Cette voie, de toute première importance, pourrait établir un lien entre facteurs de croissance, formation d'InsP $\mathrm{P}_{3}$, mouvements de $\mathrm{Ca}^{2+}$ et prolifération cellulaire [3].

En 1989, K. Mikoshiba et son groupe ont obtenu la séquence du récepteur de l'InsP $\mathrm{P}_{3}$ du cervelet de souris par clonage d'un ADNc. Le récepteur (1 MDa) de l'InsP ${ }_{3}$ est constitué de quatre sous-unités homologues d'environ $250 \mathrm{kDa}$. Plusieurs formes du récepteur, provenant d'épissage alternatif ou de gènes séparés, ont été mises en évidence [4]. Les méthodes de l'immunocytochimie ont permis de montrer que le récepteur de l'InsP $\mathrm{P}_{3}$ est localisé dans des domaines particuliers du réticulum endoplasmique, sous la membrane plasmique, à travers la cellule et dans la membrane périnucléaire du noyau [5].

Les propriétés d'ouverture et de fermeture du canal ont été décrites après reconstitution du récepteur dans des membranes artificielles. L'utilisation des méthodes de mélange et de filtration rapides ont permis de montrer que le récepteur de l'Ins $_{3}$, de différentes origines tissulaires, s'ouvre avec une cinétique très rapide, voisine de 20 à $100 \mathrm{~ms}$ [2]. D'autres études ont montré que le récepteur est réglé par le $\mathrm{Ca}^{2+}$ cytosolique. Par exemple, le récepteur de foie de rat présente trois états d'affinité différents, interconvertibles, contrôlés par le $\mathrm{Ca}^{2+}$ : deux états de basse affinité, représentant deux formes du canal ouvert, et une forme de forte affinité, représentant la forme fermée [6]. Il apparait actuellement que le récepteur est activé par les basses concentrations de $\mathrm{Ca}^{2+}(0,1$ à $1 \mu \mathrm{M})$ et inhibé par les concentrations plus élevées ( 1 à $10 \mu \mathrm{M})$. De plus, l'effet activateur du $\mathrm{Ca}^{2+}$ cytosolique est rapide, alors que l'effet inhibiteur est retardé. Il apparaît ainsi que le récepteur de l'InsP ${ }_{3}$ possède un système complexe de régulation par le $\mathrm{Ca}^{2+}$ cytosolique, voire par le $\mathrm{Ca}^{2+}$ stocké dans le réticulum endoplasmique [7], qui permet de comprendre la naissance des oscillations et la propagation des vagues $\mathrm{Ca}^{2+}$ dans les cellules stimulées par les hormones [3, 8].

Un autre mécanisme de libération du $\mathrm{Ca}^{2+}$, initialement décrit dans le muscle squelettique et le muscle cardiaque, a été ultérieurement retrouvé dans le réticulum endoplasmique de cellules non musculaires [9]. Dans un tel système, appelé $\mathrm{Ca}^{2+}$ induced $\mathrm{Ca}^{2+}$ release, une variation modérée de $\mathrm{Ca}^{2+}$ cytosolique sensibilise le canal $\mathrm{Ca}^{2+}$ et provoque une libération amplifiée de $\mathrm{Ca}^{2+}$ depuis des compartiments intracellulaires. Le canal $\mathrm{Ca}^{2+}$ est modulé par la ryanodine et la caféine. Le récepteur de la ryanodine présente des similitudes de séquences avec le récepteur de l'InsP $_{3} \quad\left(\mathrm{~m} / \mathrm{s} \quad n^{\circ} 1\right.$, vol. 6, p. 72; $n^{\circ} 4$, vol. 9, p. 465; $n^{\circ} 11$, vol. 9, p. 1278). Les différentes méthodes utilisées pour mesurer la $\left[\mathrm{Ca}^{2+}\right]_{i}$ dans les cellules vivantes (cellules en suspensions, cellules uniques ou systèmes multicellulaires en primocultures) sont rapportées dans l'article de C. Pavoine et F. Pecker. Les indicateurs utilisés sont des protéines bioluminescentes [1], ou des indicateurs fluorescents[10]. La méthode du path-clamp sur cellule entière $[11,12]$ a également été très utilisée par l'enregistrement de courants ioniques dépendant de la $\left[\mathrm{Ca}^{2+}\right]_{i}$. Cette dernière méthode autorise la perfusion en continu du cytosol à travers la pipette de patch et ainsi, l'accès à la face interne de la membrane plasmique pendant l'expérimentation (diffusion de seconds messagers, en particulier).

Comme nous l'avons mentionné dans l'introduction, les hormones déclenchent des cycles lents (1 pic par min) d'oscillations de la $\left[\mathrm{Ca}^{2+}\right]_{i}[1]$. La fréquence des pics dépend de la concentration d'hormones. Le retrait du $\mathrm{Ca}^{2+}$ extracellulaire ralentit, puis bloque les oscillations, du fait de la décharge progressive, puis complète, du réticulum endoplasmique. Cela confirme le rôle majeur des compartiments intracellulaires dans l'initiation des oscillations, et le rôle de soutien apporté par l'influx de $\mathrm{Ca}^{2+}$ extracellulaire. Dans le foie entier, stimulé par des hormones, on observe une libération oscillante de $\mathrm{Ca}^{2+}[13]$, ce qui implique une coordination dans l'organe entier des oscillateurs présents dans chaque cellule. Certains modèles proposent que les oscillations de la $\left[\mathrm{Ca}^{2+}\right]_{i}$ résulteraient d'oscillations primaires de la production d'InsP $\mathrm{P}_{3}[1]$. D'autres décrivent les oscillations de $\left[\mathrm{Ca}^{2+}\right]_{i}$ à concentration constante d'InsP. ${ }_{3 .}$ Pour ces derniers, les oscillations découleraient des propriétés du récepteur de l'InsP ${ }_{3}$, activé ou inhibé par le $\mathrm{Ca}^{2+}$, décrites cidessus, qui pourraient entrainer des cycles de libération et de réaccumulation du $\mathrm{Ca}^{2+}$ du réticulum endoplasmique [3].

Les oscillations présentent des avantages évidents pour les cellules. En premier lieu, elles évitent les montées de $\left[\mathrm{Ca}^{2+}\right]_{\mathrm{i}}$ prolongées qui entraîneraient inévitablement des dommages cellulaires irréversibles en activant les phosphatases et les protéases cellulaires. De plus, les augmentations cycliques de la $\left[\mathrm{Ca}^{2+}\right]_{i}$ préviennent la perte d'énergie induite par la stimulation excessive de multiples systèmes consomma- 
teurs d'ATP, tels que les kinases, les transporteurs et les ATPases. Enfin, elles évitent la désensibilisation des systèmes effecteurs, induite par les élévations soutenues de $\left[\mathrm{Ca}^{2+}\right]$. Ainsi, l'augmentation de la $\left[\mathrm{Ca}^{2+}\right]_{i}$ pendant un temps court peut être suffisante pour stimuler les protéines sensibles au $\mathrm{Ca}^{2+}$ de façon maximale, sans risque de mise en route des mécanismes de désensibilisation. Les techniques de numérisation de l'image en vidéomicroscopie ont permis de suivre les variations de la $\left[\mathrm{Ca}^{2+}\right]$ à une échelle subcellulaire, avec une bonne résolution spatiale et temporelle [10]. Elles sont décrites dans l'article de C. Pavoine et F. Pecker. Ces analyses ont montré que l'application d'une hormone peut entraîner des montées de la $\left[\mathrm{Ca}^{2+}\right]_{\text {i }}$ sous la membrane plasmique de lá cellule. Dans certaines conditions, cette concentration élevée de la $\mathrm{Ca}^{2+}$ peut rester localisée et activer des protéines sensibles au $\mathrm{Ca}^{2+}$ (canaux ioniques, transporteurs, enzymes, etc.) dans cette région particulière de la cellule [14]. Dans d'autres conditions, le front du gradient de $\mathrm{Ca}^{2+}$ se propage avec des vitesses de l'ordre de quelques dizaines de $\mu \mathrm{m} / \mathrm{s}$ dans l'ensemble de la cellule sous la forme d'une vague $\mathrm{Ca}^{2+}$. Le $\mathrm{Ca}^{2+}$ peut alors activer des protéines localisées dans d'autres endroits de la cellule. Quand des cellules sont associées, ces vagues peuvent se propager de cellules en cellules via les jonctions communiquantes (gap junctions) [14]. On a proposé que le $\mathrm{Ca}^{2+}$ libéré par $\mathrm{I}^{\prime} \mathrm{InsP}_{3}$ dans une région localisée sous la membrane plasmique pourrait successivement sensibiliser d'autres récepteurs de l'InsP ${ }_{3}$ plus profonds et moins sensibles [3]. Une autre possibilité est que la vague de $\mathrm{Ca}^{2+}$ serait due à une vague de production d'Ins $\mathrm{P}_{3}$ débutant au niveau d'un site de la membrane plasmique et se propageant à l'ensemble de la cellule [8].

L'universalité des oscillations dans les cellules animales, et le fait qu'elles soient associées à des varia- tions localisées ou propagées de la $\left[\mathrm{Ca}^{2+}\right]_{i}$ conduisent la plupart des groupes de recherche à imaginer que ces vagues oscillantes sont une propriété intrinsèque de beaucoup d'organes, et qu'elles constituent un mode général de codage de la réponse hormonale [3]. Le rôle éventuel de cette voie InsP $_{3}-\mathrm{Ca}^{2+}$ dans certaines autres fonctions cellulaires reste à établir. Par exemple, l'existence d'un transport de $\mathrm{Ca}^{2+}$, d'une phospholipase C- $\beta$ et de récepteurs de l'InsP ${ }_{3}$ dans le noyau des cellules de mammifères n'a toujours pas permis d'établir le rôle de cette voie de signalisation dans l'organite (m/s $n^{\circ} 1$, vol. 1), p. 123). Un autre exemple est celui du rôle des facteurs de croissance, qui stimulent la production d'Ins $\mathrm{P}_{3}$, dans la régulation de la croissance cellulaire. Il est possible que la voie $\mathrm{InsP}_{3}-\mathrm{Ca}^{2+}$ soit impliquée dans la séquence des événements qui se terminent par une synthèse d'ADN dans certaines cellules [3].

\section{RÉFÉRENCES}

1. Cobbold PH, Sanchezbueno A, Dixon CJ. The hepatocyte calcium oscillator. Cell Calcium 1991 ; 12 : 87-95.

2. Tran D, Noèl J, Claret M. Calcium et foie. Arch Int Physiol Biochem Biophys 1993; 101: 23-40.

3. Berridge MJ. Inositol trisphosphate and calcium signalling. Nature 1993; 361: 315-25.

4. Mikoshiba $\mathrm{K}$ Inositol 1,4,5-trisphosphate receptor. Trends Physiol Sci 1993 ; 14 : 86-9.

5. Meldolesi J, Madeddu L, Pozzan T. Intracellular $\mathrm{Ca}^{2+}$ storage organelles in non-muscle cells : heterogeneity and functional assignment. Biochim Biophys Acta 199(); 1055: 13()-40.

6. Pietri-Rouxel F, Hilly M, Mauger JP. Characterization of a rapidly dissociating inositol 1,4,5-trisphosphate-binding site in liver membranes. / Biol Chem. 1992: 267 : 20)17-23.

7. Irvine RF. Inositol tetrakisphosphate as a second messenger. (onfusions, contradictions and a potential resolution. Biokssays $1991 ; 13: 419-27$

8. Meyer T. Cell signaling by second messenger waves. (iell 1991; 64: 675-8.

9. Galione A, Lee HC, Busa WB. $\mathrm{Ca}^{2+}$-induced $\mathrm{Ca}^{2+}$ release in sea urchin egg homogenates-modulation by cyclic ADP-ribosome. Science 1991; 253 : 1143-6.

10. Tsien RY. Intracellular signal transduction in four dimensions: from molecular design to physiology. Am / Physiol 1992; 32 : $723-8$.

11. Neher E. Ion channels for communication between and within cells. Science 1992 ; 256 : 498-5()2.

12. Sauvé R. Étude électrophysiologique des canaux ioniques isolés. médecine/sciences $1991 ; 7: 985-6$

13. Graf P, Von Dahl S, Sies H. Sustained oscillations in extracellular calcium concentration upon hormonal stimulation of perfused rat liver. Biochem / 1987; 241 : 933-6.

14. Sanderson MJ, Charles AC, Boitano S, Dirksen ER. Mechanisms and function of intracellular calcium signaling. Mol Cell Endocrinol 1993; 98 : 173-87.

TIRÉS A PART

M. Claret. 